\title{
Acertando o passo! Falar de deficiência mental é um erro: deVe FALAR-SE DE DIFICULDADE INTELECTUAL E DESENVOLVIMENTAL (DID). POR QUÊ?
}

THE TERM "MENTAL RETARDATION" IS A Mistake: WHY NOT "INTELLECTUAL and DeVelopmental Disability": CONCEPTUal and PORTuguese linguistic CONSIDERATIONS?

Sofia SANTOS ${ }^{1}$

Pedro MORATO

\begin{abstract}
RESUMO: a nova concepção e terminologia adotada pela língua inglesa no contexto da ainda designada "deficiência mental" parece ter alcançado um consenso estável e satisfatório face às expectativas que a sociedade deve esperar das populações com esse diagnóstico, enfatizando agora o impacto que as exigências do envolvimento detém no desenvolvimento individual, reforçando-se, assim, a necessidade emergente da alteração de mentalidades e atitudes para com estas populações. Para este efeito, o presente artigo introduz uma nova proposta de alteração da terminologia em português de deficiência mental/intelectual para "dificuldade intelectual e desenvolvimental", explicitando e fundamentando as razões para a mesma.
\end{abstract}

PALAVRAS-CHAVE: Educação Especial. Terminologia. Deficiência Intelectual. Dificuldade intelectual.

\begin{abstract}
The new conception and terminology of "intellectual disability" adopted in the English language referring to what was known earlier as "mental retardation" seems to have achieved a satisfactory and stable consensus regarding society expectations for those groups to which this diagnosis refers. Currently, the focus is on the environmental impact on human functioning and development, which will reinforce the emergent need for altering attitudes towards such groups. To this end, this article defends a new proposal changing the Portuguese term from mental retardation to intellectual and developmental disability, establishing the term's meaning and boundaries.
\end{abstract}

KEYWORDS: Special Education. Terminology. Intellectual Disability.

\section{INTRODUÇÃo}

Estamos em pleno século XXI! E no entanto, ao analisar os novos avanços no estudo das deficiências, é facilmente constatável a ainda existente discussão intensa no campo das pessoas que apresentam características diferentes quando comparadas com os valores normativos e expectáveis. E quando a discussão entre na esfera da até agora denominada Deficiência Mental, onde a própria terminologia parece trazer consigo alguma descredibilização sobre estas populações, tudo parece assumir proporções ainda maiores!

\footnotetext{
${ }^{1}$ Doutorada em Motricidade Humana na especialidade de Educação Especial, Centro de Estudo Interdisciplinar da Performance Humana, Departamento de Educação Especial e Reabilitação, Faculdade de Motricidade Humana, Universidade Técnica de Lisboa. sofiasantos@fmh.utl.pt

${ }^{2}$ Doutorado em Motricidade Humana na especialidade de Educação Especial, Centro de Estudo Interdisciplinar da Performance Humana, Departamento de Educação Especial e Reabilitação, Faculdade de Motricidade Humana, Universidade Técnica de Lisboa. pmorato@fmh.utl.pt
} 
Esta mesma revolução e tentativa de mudança torna-se, agora, emergente e fulcral no seio da investigação mais atual que se faz sentir na abordagem à condição em questão, no âmbito do seu construto e respectiva terminologia. E é também neste meio que, apesar desta evolução, ainda se considera difícil responder à questão sobre "qual a melhor maneira de se fazer referência aos indivíduos que enfrentam algum grau de limitação ou restrição funcional" (OMS, 2004, p. 215).

Por outro lado, "os termos são a designação de conceitos definidos em expressões linguísticas, tais como, palavras ou frases" (OMS, 2004). Neste âmbito e de acordo com Schalock et al. (2007a e 2007b), assiste-se agora a uma mudança de paradigma, onde a renomeação da ex-Deficiência Mental se ergue como uma necessidade imperiosa. Neste sentido, os autores clarificam o conceito e procuram que a nova terminologia seja o reflexo das características da sua definição, pelo que enfatizam a pertinência de uma reflexão crítica e matura sobre os termos apropriados a adotar.

Da mesma opinião, a OMS (2004) advoga que, no momento atual de vida, é fundamental que se chegue a um acordo entre os termos a utilizar em cada idioma, avançando com os adjetivos de precisão, aceitabilidade e utilidade geral. Desta mesma opinião são Amiralian et al. (2000) para quem as "[...] imprecisões [...] resultam na dificuldades na aplicação e utilização do conhecimento produzido."

De acordo com as investigações e reflexões científicas mais recentes (GOODE, 2002; LUCKASSON et al, 2002; MORATO; SANTOS, 2007; PANEK; SMITH, 2005; SCHALOCK et al, 2007a; SCHALOCK et al, 2007b; SCHALOCK et al, 2010) parece que a nova definição e a nova terminologia ganharam já um consenso em inglês, adoptando-se a expressão intellectual disability, apesar de na opinião de Walsh (2002) o termo cognitive-adaptive disability ser o mais próximo dos critérios utilizados para a avaliação e diagnóstico da condição.

E no caso dos países onde se fala português? Parece que ainda não foi encontrada uma solução consolidada, constatando-se a ainda existência de variadas nomenclaturas (deficiência mental, atraso mental, incapacidade mental...). Como é que nos estamos a colocar perante todas as inovações sentidas neste âmbito?

Antes de avançar (com mais análise de reflexão), há que assinalar que o grande objetivo deste artigo é que a adoção de uma nova terminologia, em consonância com o modelo conceitual de base, produza mudanças significativas na forma de estar e de trabalhar com o diagnóstico (identificação) em análise: isto é, novas perspectivas (com base nos apoios e não nos déficits/pessoas) e novos sistemas de avaliação (dos apoios) que contribuirão para a planificação e implementação de intervenções mais pertinentes e individualizadas.

Segundo Luckasson e Reeve (2001) existem cinco fatores importantes que devem ser considerados na escolha de um novo nome, ou seja, uma nova identificação, que deverá:

1. ser específica permitindo a sua diferenciação com outras entidades; 
2. ser consistente na sua utilização;

3. incorporar o conhecimento mais atualizado no âmbito científico e profissional;

4. ser robusta na sua operacionalização de forma a ser utilizada para diferentes decisões (diagnóstico, classificação, planejamento...); e

5. refletir a componente essencial da nomeação de um grupo específico, comunicando valores importantes de respeito e dignificação (vs estigmatização e rotulagem).

Num artigo sobre a questão da nomenclatura Smith (2002), cita Kuhn (1962) quando este afirmava que as perspectivas do momento se vão tornando tão enraizadas e institucionalizadas que apenas uma quebra dramática e repentina das ideias mais convencionais poderia trazer uma revolução positiva ao nível da reflexão e pensamento.

Nesta sequência, a atual mudança do paradigma nesta área terá profundas implicações no âmbito da saúde, educação, segurança social sendo, atualmente, cada vez mais confrontados com a necessidade de processos mais atuais e contextualizados, menos estigmatizantes e mais funcionais, de olhar para as características desta problemática.

As novas perspectivas que agora emergem redirecionam-se para $\mathrm{o}$ paradigma mais ecológico, onde a valorização do envolvimento se torna uma realidade com impacto no desenvolvimento individual, havendo agora uma atenção específica na qualidade das respostas das pessoas (com e sem DID) às exigências dos fatores ambientais com as quais se vão confrontando diariamente. Tal como referido por Omote (1996 apud COSTA, 2010) a definição de, então denominada, deficiência mental passa a situar-se num contexto temporal, espacial e socialmente determinado, havendo necessidade de se especificar os critérios e, acrescentamos nós, uma nova designação.

Tal como Morato eSantos (2007) afirmam, a referência científica à deficiência mental tem vindo a ser questionada, do ponto de vista conceitual, como pouco rigorosa, pois, desde sempre, a maturidade social e o comportamento adaptativo eram apontados como critérios de diagnóstico (identificação), não tendo sido, no entanto, formalmente utilizados. E tal como é facilmente constatável, a concepção da adaptação, a provisão de uma rede de apoios para a vivência de uma vida independente são agora as palavras de ordem em todas as intervenções no campo.

Antes de serem apresentados uma série de reflexões teóricas e práticas sobre o tema que explicarão e contextualizarão melhor a nossa proposta, deixamos desde já o novo termo que nos parece ser o mais adequado (correto) ao momento atual: Dificuldade Intelectual e Desenvolvimental (DID), passando então a ser utilizada esta nova designação para nos referirmos a todos os diagnósticos (ou identificações) até agora e anteriormente conhecidos por deficiência mental! Ao 
longo do documento (deste texto) irão ser enumeradas as várias razões sobre as quais se baseia esta proposta.

\section{A Universalidade DA TERMinOlogia}

Os construtos e a sua definição-compreensão são moldados pelas políticas sociais e pelo contexto sociocultural onde os sujeitos se inserem (SMITH, 2002). É no seio desta ideia que Mazzotta (2008) afirma que a "compreensão dos princípios e ações estão imbricados de tal forma que os princípios somente decorrem [...] na sociedade enquanto duram as ações que deles decorrem”. E o que é possível constatar é que qualquer que tenha sido o nome adotado no passado pela condição em reflexão, traduzia-se sempre num olhar negativo, pejorativo, desconhecido, minimizado... (SMITH, 2002).

Todo este conjunto de ideias vem expresso no novo documento produzido pela OMS $(2001,2004)$ que na opinião de Diniz, Medeiros e Squinca (2007) representa uma tentativa internacional de organizar uma linguagem universal para redefinir a deficiência agora de uma categoria biomédica, para um modelo biopsicosocial.

Esta classificação de funcionalidade deixa cair o termo incapacidade e apesar de ainda o utilizar ao longo do documento, os autores alertam, no anexo 5, que a "OMS reconhece, em particular, que os termos utilizados na classificação podem, apesar de todos os esforços, estigmatizar e rotular" (p. 215). Por outro lado, apela igualmente a uma nova perspectiva sobre a pessoa, relevando a participação de qualquer pessoa em permanente interação com os fatores ambientais (OMS, 2004). A funcionalidade é agora a palavra-chave no campo das populações especiais, onde realmente o fócus redireciona-se, à luz das mais recentes investigações, para a qualidade de interação dinâmica e de participação nas atividades expectáveis para qualquer cidadão da comunidade onde se insere, relegando para segundo plano a questão de centrar-se exclusivamente no problema da pessoa.

A funcionalidade é como um termo genérico indicador dos aspectos positivos da interação entre o indivíduo e fatores contextuais e a incapacidade como um conjunto de aspectos negativos desta interação (OMS, 2004, p. 186). As componentes da funcionalidade podem, então, ser expressos de duas maneiras: para indicar problemas (incapacidade, limitação da atividade e restrição na participação designadas pelo termo genérico de deficiência) e outra para aspectos não problemáticos sob o termo de funcionalidade (p. 12). Ou seja, começam a aparecer termos como limitação, dificuldades ou restrições, com caráter de possibilidade de recuperabilidade (com os apoios corretos) em detrimento da utilização de palavras mais rotulativas e estigmatizantes como a incapacidade. É possível constatar, que no mesmo documento "limitações na atividade" são entendidas como dificuldades na execução das atividades.

Diniz, Medeiros e Squinca (2007) reforçam esta ideia, afirmando que a causalidade entre impairments, disabilities e handicaps conduziria a situações de 
restrições de participação que, por sua vez, fariam com que a pessoa apresentasse uma desvantagem social, reforçando mais uma vez o impacto que o envolvimento tem na forma de perspectivar as populações diagnosticadas com a condição em questão.

Ainda no mesmo documento, deficiência é definida por "problemas nas funções ou estruturas do corpo" (OMS, 2004, p. 13) e reporta-se a valoresdesvios do que é considerada a norma, relembrando-se que este desvios podem ser temporários, intermitentes... podendo variar ao longo do tempo e não se mantendo imutável, tal como a designação de incapacidade e deficiência mental podem induzir.

Neste âmbito, Morato e Santos (2007) avançam com a ideia de que a palavra deficiência é mais estigmatizante, dado inferir-se da mesma a noção de imperfeito ou com déficits, termos que na língua portuguesa denotam um caráter negativo e pejorativo. Esta imperfeição, e no momento atual de vida, está associado à irrecuperabilidade e inutilidade das pessoas designadas como "deficientes", assumindo assim um enorme impacto social negativo.

De qualquer das formas, a responsabilidade de ser portador de deficiência não é mais encarada como exclusiva do indivíduo, considerando-se igualmente a importância da influência exercida pelo envolvimento e pelos tipos de apoios prestados: a expressão da interação - capacidades, envolvimento e funcionalidade (LUCKASSON et al.; SMITH, 2003; SCHALOCK et al. 2007a; SCHALOCK et a., 2010).

Diniz, Medeiros e Squinca (2007) corroboram esta ideia afirmando que "[...] a experiência da desigualdade pela deficiência resultava mais de estruturas sociais pouco sensíveis à diversidade que de um corpo com lesões" (p. 2508).

Ao longo de toda a pesquisa bibliográfica é fácil encontrar várias terminologias para designar as mesmas condições, variando as mesmas com o tempo e o conhecimento da condição, e com a diferenciação cultural de cada país.

Diniz, Medeiros e Squinca (2007) continuam, apontando como exemplo, a adoção da terminologia de necessidades educativas especiais no âmbito da educação pelo Reino Unido, realçando a proposta mais inovadora e atual da classificação (LUCKASSON et al., 2002;SCHALOCKet al.,2010), não pelo déficit, mas antes pelo tipo de apoios individuais necessários para a aquisição do funcionamento independente

Neste momento, parece oportuno então apresentar a nova definição vigente de DID, cuja primeira grande mudança se centra agora na qualidade de interação entre o sujeito e o seu envolvimento, em detrimento de se ocupar exclusivamente dos déficits individuais. Por outro lado, são ainda acrescentados pressupostos de que todos os indivíduos, independentemente do seu rótulo têm áreas fortes e menos fortes e que com um sistema de apoios adequados e individualizados o sujeito com DID irá assumir o seu papel como cidadão ativo, tomando as decisões pessoais relativas à sua própria vivência, contribuindo para a melhoria da qualidade de vida (LUCKASSON et al., 2002; SCHALOCK et al., 2010). A ênfase é assim, colocado no padrão dos apoios necessários... 
É então tempo para admitir que o termo até agora utilizado - deficiência mental - não detém qualquer valor científico, sendo socialmente estigmatizante, ao mesmo tempo que, para vários autores a expressão se mantém confusa e insatisfatória (SMITH, 2002; SMITH, 2003; LUCKASSON et al, 2002). Também para Leonard e Wen (2002) "a definição, a classificação e avaliação da deficiência mental" tem sido alvo de grande controvérsia, ao longo dos tempos. É tempo para encarar a realidade e começar a fazer a diferença.

Corroborando esta ideia Yuker (1988 in PANEK; SMITH, 2005) concluiu, no seu estudo sobre disability hierarchy, e utilizando a técnica de diferenciação semântica, que o termo anterior de mental retardation (traduzido na altura por deficiência mental) ocupava o $22^{\circ}$ lugar (entre 24 designações) no ranking de aceitabilidade, isto é, de 24 termos de diversos diagnósticos que variavam do (ou menos) não-estigmatizantes (por exemplo, diabetes) ao mais estigmatizante, o termo deficiência mental parecia imbuído de fraca consideração (apenas suplantado pela multideficiência e condições afins).

Pegando nas palavras de Wolfensberger (2002), teremos que ser inteligentes o suficiente para que com a nova terminologia se inicie toda uma nova forma de pensar a pessoa com esta condição. O termo a adotar deverá então remover a carga negativa que a sociedade ainda detém face a esta problemática, esperando com isto mudar de forma positiva as atitudes e as expectativas face à classe de pessoas em questão. Neste sentido, os termos a designar deverão ser realistas e positivos, não degradando a condição humana.

Seguindo as diretrizes semânticas de Wolfensberger (2002), o novo nome não deverá poder ser convertido num verbo ou num nome (deficiente mental, incapaz mental...), ao mesmo tempo que, quando se referir à pessoa a transformação do português seja mais do gênero a pessoa com dificuldades... do que pessoa deficiente ou incapaz... Neste campo, muitos autores avançam inclusive com exemplos onde o nome se transforma num adjetivo pejorativo ou insultuoso (Deficiência Mental ou Incapacidade Mental = ...és um incapaz! Ou ...és um deficiente!).

Também no anexo 5 da CIF (OMS, 2004) se explicita que: "em vez de se referir a uma pessoa mentalmente incapacitada, a classificação utiliza a frase "pessoa com um problema de aprendizagem".

Concordando com esta lógica, Walsh (2002) e Gelb (2002) acrescentam ainda que a utilização coloquial de deficiência mental banalizou-se, tornando-se um insulto nos meios escolar e de comunicação, sendo utilizados como adjetivos pejorativos e ofensivos.

Por outro lado, Wolfensberger (2002) avança ainda com a ideia de quando um termo adquire uma imagem negativa, a necessidade de alteração do mesmo, justifica-se, apenas se o novo termo for considerado como uma melhoria, não se desviando da definição subjacente a um determinado diagnóstico, não só ao nível da imagem como também dos respectivos critérios. Walsh (2002) acrescenta 
inda que os construtos andam de mãos dadas com a linguagem e que por isso, a designação de um novo termo é realmente importante!

Também Schalock et al. (2010) recomendam um repensar na abordagem deste diagnóstico, advogando uma postura mais ecológica e centrada nos apoios (THOMPSON et al, 2004; SANTOS et al, 2008), traduzindo disability como a expressão de limitações (no original, expression of limitations in...) e, ao contrário de algumas correntes de pensamento, não de incapacidades, que sugere por si só a noção de ser incapaz e onde parece estar subjacente a questão de ser uma situação permanente. Também Baroff (2003) defende a adoção de uma terminologia que caracterize a condição, apelando às qualidades e não aos déficits/estigmas.

$\mathrm{Na}$ Inglaterra, e de acordo com o White Paper Valuing People (DEPARTMENT OF HEALTH, 2001) a condição de learning disabilities é definida com os mesmos três critérios presentes na definição da AAIDD (LUCKASSON et al., 2002; SCHALOCK et al., 2010) e da ICD-10 (WHO, 1996) de intellectual disabilities e onde se incluem as limitações ao nível do funcionamento cognitivo, do funcionamento social ou comportamento adaptativo, adotando os 18 anos como idade-teto para a sua identificação. O mesmo documento ressalva ainda que apesar da utilização do termo ofensivo de mental retardation pelo documento da OMS, em Inglaterra o termo adotado será, então, o de learning disabilities.

De uma forma geral e porque parece ser consensual (MORATO; SANTOS, 2007; ROSSATO; LEONARDO, 2011; SASSAKI, 2005; VERDUGO; SCHALOCK, 2010) o termo intelectual não parece denotar qualquer controvérsia e por isso a reflexão incidirá mais na outra parcela da expressão: deficiência ou dificuldade?

Ao analisar as diferentes traduções utilizadas em Português, é fácil perceber a inexistência de uma coerência, dado utilizarem-se diferentes palavras para a mesma palavra original (Intellectual Disability é traduzida como Deficiência Intelectual; e Learning Disability como Dificuldades de Aprendizagem).

Também é possível perceber que o termo dificuldade está em permanente associação com as limitações presentes na definição de DID, estando-lhe inerente a premissa de que com os apoios adequados essa dificuldade pode ser minimizada e mesmo ser ultrapassada, aumentando ou diminuindo em função da manipulação do meio (SANTOS; MORATO, 2002), estando-lhe subjacente uma expectativa mais positiva do que o termo deficiência (MORATO; SANTOS, 2007) ou incapacidade (SASSAKI, 2005).

Assim, no estudo concretizado por Rossato e Leonardo (2011), cujo objetivo era de compreender quais as expectativas e concepções oferecidas no seio da educação especial, foi possível verificar que em $44 \%$ da amostra o índice mais elevado de resposta (entre uma série de possíveis opções) foi que "as dificuldades de aprendizagem é sanada e a deficiência intelectual é definitiva" (p. 79). Os autores prosseguem com possíveis explicações, avançando com as hipóteses de que o termo "deficiência remete para algo estático, irreversível, instalado, sem possibilidade 
de desenvolvimento", enquanto que o termo "dificuldade" é entendido como possibilidade de "superação [...] por seu caráter momentâneo e dinâmico" (p. 79).

Os mesmos autores avançam com uma explicação para esta escolha, colocando a possibilidade da mesma evidenciar falta de perspectiva de desenvolvimento destas populações, restringindo-se à condição biológica, excluindo a questão social e adaptativa.

De fato há que concordar com Rossato e Leonardo (2010) quando, face a estes resultados, se questionam se os professores têm esta concepção da condição de DID, então de que forma e que conteúdos são transmitidos aos estudantes com DID em ambiente educativo? E que resultados são esperados destes indivíduos "Como [...] se sentem ao olhar, todos os dias, para seu aluno e ver nele todos esses atributos relegados à deficiência?" (p. 81). Esta dúvida remete, mais uma vez, para a necessidade de redefinição do conceito e da terminologia que se tenta advogar ao longo deste documento.

Se nos guiarmos pela incapacidade, implícita às atuais terminologias da condição em análise, como fator determinante das características destes sujeitos estaremos a ter um papel ativo na exclusão dessas mesmas pessoas, enquanto que ao adotarmos terminologias mais consistentes com as novas concepções e paradigmas, como o caso de Dificuldade Intelectual e Desenvolvimental, estaremos a modificar pensamentos, que por sua vez se irão repercutir ao nível das planificações das intervenções e, finalmente, alterar expectativas e perceber-se o direito de qualquer pessoa (com ou sem diagnóstico) em assumir o seu papel como cidadão ativo.

Por outro lado, e no âmbito das nomenclaturas presentes no seio das populações especiais, observa-se a existência de utilizações de expressões como Dificuldades de Aprendizagem como tradução direta do Learning Disabilities, o que foi facilmente aceite e consensual quer como nome, quer como construto.

Assim, não tem qualquer sentido complicar esta aparente ambiguidade em desfavor do rigor, mas entender esse rigor na coerência intelectual e linguística que entre nós se desenvolveu e agora o que nos espera perante a condição de DID é ser-se capaz de explicar em coerência a sua utilização que é, de facto, substantiva e significativa (MORATO; SANTOS, 2007).

Esta ideia é corroborada por Rossato e Leonardo (2011) que afirmam que "a mudança dos termos está mais ligada à realidade e necessidade histórica" (p. 72).

A afirmação do documento da OMS e as novas políticas sociais advogadas pela AAIDD permitem o reconhecimento da força política do modelo social (DINIZ, MEDEIROS; SQUINCA, 2007) cujo fócus é colocado agora na (qualidade) de interação entre qualquer sujeito e as exigências do envolvimento onde se insere e cresce como ser humano.

Do ponto de vista prático, e nesta nova filosofia adaptativa e social, torna-se claro que os termos "deficiência mental" e "incapacidade mental" não se 
relacionam de forma alguma com a definição sobre a qual baseamos toda a política portuguesa e que implica a concomitância de limitações intelectuais e adaptativas que se expressam nas habilidades conceptuais, práticas e sociais (LUCKASSON et al., 2002; SCHALOCK et al., 2010).

Ao analisar as intervenções na área desta população, é possível verificar que as mesmas se direcionam para a provisão de apoios nas áreas do comportamento adaptativo e funcionalidade e não estritamente para as "habilidades ou capacidades intelectuais"- WALSH, 2002).

A nova definição e abordagem de DID adotada desde 2002 (LUCKASSON et al., 2002), que comporta em si a noção de "habilidades" adaptativas, estabelece que o diagnóstico da condição em questão passa a ser perspectivada numa relação sistemática e permanente com o comportamento adaptativo, com repercussões fulcrais em termos de avaliação, intervenção, e inclusive, escolarização. Desta forma, a DID é entendida como o resultado da existência de limitações ao nível do funcionamento intelectual concomitantes com as limitações ao nível das competências adaptativas antes dos 18 anos e que se expressam nas habilidades práticas, cognitivas e sociais.

Para Schalock (2003), este reconhecimento da coexistência de déficits nas áreas da competência social, prática e conceptual, permite reequilibrar a área cognitiva com a social e instrumental (e não se basear exclusivamente naquela), na interação diária com o envolvimento, num processo de avaliação holístico e sistêmico do indivíduo.

Esta nova perspectiva vem contrariar a tendência anterior em se "rotular e estigmatizar", classificando-se os indivíduos em vários graus de deficiência mental: leve, moderado, severo e profundo. Atualmente, Luckasson et al. (2002) "classificam" os indivíduos com DID, não pelas características intrínsecas aos mesmos, mas pelo tipo de apoios que necessitam para ultrapassar as suas dificuldades: 1) apoios intermitentes - necessários esporadicamente (natureza episódica e descontínua), na medida em que o sujeito nem sempre necessita do mesmo ou apenas necessita em períodos específicos de transição, podendo ser de alta ou baixa intensidade; 2) apoios limitados - caracterizados por uma certa consistência em termos de intensidade (natureza contínua), especialmente nos períodos críticos; 3) apoios extensivos - que denotam já um acompanhamento regular (diário) pelo menos nalguns contextos específicos (casa, escola, trabalho...), não se encontrando definido o tempo da sua aplicação; e finalmente, 4) apoios permanentes - caracterizados pela sua constância e altas intensidades, de estilo permanente e denotando uma maior intrusividade do que os restantes.

Tal como vem advogado na CIF (OMS, 2004, p.8), as informações sobre o diagnóstico e a funcionalidade de uma pessoa trarão consigo uma imagem mais ampla e significativa da saúde da população, que podem e devem ser consideradas nas tomadas de decisão, que deterão consequências funcionais e sociais para todos. 
Concluindo, eis então algumas das principais razões para a adoção do termo Dificuldade Intelectual e Desenvolvimental (VERDUGO; SCHALOCK, 2010):

1. evidencia o construto socioecológico atual (LUCKASSON et al, 2002; SCHALOCK et al., 2010) cuja relação indivíduo-envolvimento assume o papel principal, "demarcando-se do postulado psicométrico absolutista e imperialista" (MORATO; SANTOS, 2007);

2. permite um alinhamento consistente com as práticas profissionais atuais centradas mais na questão do comportamento adaptativo e da funcionalidade (VERDUGO; SCHALOCK, 2010);

3. é compatível com a mais recente proposta de classificação (LUCKASSON et al., 2002; SCHALOCK et al., 2010) baseada na necessidade de apoios (em detrimento );

4. que assenta no novo paradigma de apoios que assume que com os apoios adequados a qualidade de vida de cada um melhorará (MORATO; SANTOS, 2007; THOMPSON et al, 2008; VERDUGO; SCHALOCK, 2010)

5. é menos ofensivo e estigmatizante (MORATO; SANTOS, 2007; VERDUGO; SCHALOCK, 2010).

A opção do termo intelectual, pelo anterior de mental, parece realmente não ser tão passível de discussão, verificando-se consenso nesta questão, não só porque o intelectual se refere a um conjunto de fatores subjacentes ao funcionamento da inteligência e avaliados pelos vários instrumentos de avaliação (fatores espaciais, numéricos, verbais...); que é mais analítico do que o termo mental que indicia o funcionamento da mente como um todo (MORATO; SANTOS, 2007; SASSAKI, 2005). Além disso, esta nova designação permitirá um esclarecimento da confusão, geralmente associada à distinção entre "deficiência mental" e "doença mental" (SASSAKI, 2005). Tal mudança é bem perceptível na alteração (ainda relativamente) recente do nome de inúmeras organizações (por exemplo, American Association on Intellectual Disability, Confederación Española de Organizaciones en favor de Personas com Discapacidad Intelectual, entre outros).

A associação do termo desenvolvimental explica-se pela tentativa de se expressar uma maior abrangência no que concerne às características sócioadaptativas e respectiva validade ecológica (MORATO; SANTOS, 2007).

\section{CONCLUSÃo}

"Ao mesmo tempo em que o mundo vai mudando, as nossas palavras e as nossas perspectivas e pontos de vistas também se vão alternado, e a forma como as utilizamos para descrever o mundo também se vai adaptando" (WALSH, 2002, p. 71). É então, neste clima de mudança cultural, uma mudança de linguagem e uma mudança de referenciais que vamos constatando a necessidade de alterar, igualmente, os novos termos utilizados até a este momento. 
De acordo com a OMS (2004), e reiterando a ideia de Diniz, Medeiros e Squinca (2007), encontramo-nos numa fase de desenvolvimento na qual se sente a necessidade emergente de "um acordo sobre o termo que melhor reflete o conteúdo" (p.2508) em cada idioma, ao mesmo tempo em que se deve considerar não só a escolha adequada dos termos, como também a coerência com o debate político-social atual.

Baseados nesta ideia, e até considerando a opinião de vários autores que afirmam que possivelmente qualquer "nova" nomenclatura aceite agora como menos estigmatizante, sê-lo-à considerado rotulativo daqui a uma ou duas décadas (SMITH, 2002; WALSH, 2002).

Corroborando ainda a ideia de Diniz, Medeiros e Squinca (2007), voltamos a reiterar a ideia de que o termo em português de incapacidade "ignora trinta anos de debate acadêmico e político internacional, em especial as contribuições do modelo biopsicosocial". Também para Sassaki (2005) ao conceito de incapacidade está implícito "o estado negativo de funcionamento da pessoa" (p. 9)

Tal como advogado por Santos e Morato (2010), a mudança de terminologia é uma necessidade emergente no contexto das populações até agora identificadas e classificadas como tendo Deficiência Mental, e deverá considerar um nome que identifique as características da problemática, a sua funcionalidade (e não incapacidade) que tenha em consideração que as limitações podem variar de sujeito para sujeito, ao mesmo tempo em que seja o menos estigmatizante possível. E, de acordo com as teorias, mais atuais, evidenciando a necessidade de apoios (e não dos déficits), tendo como subjacente a ideia de evolução e capacitação (vs. incapacidade - GELB, 2002; TURNBULL et al, 2002; BAROFF, 2003; GLIDDEN, 2003). Da mesma opinião, Snell e Voorhees (2002), reforçam a necessidade de uma nova nomenclatura com uma conotação mais positiva! e Baroff (2003) acrescenta que "labels cannot erase biologically determined differences, [but] the connotations that they evoke can affect the means through which those differences can be diminished". Com a nova nomenclatura esta duplicação do sentido seria, senão eliminada, pelo menos minimizada!

Também Rossato e Leonardo (2011) questionam se o diagnóstico de "deficiência mental/intelectual", e porque parece ainda deter conotações de imutabilidade e irreversibilidade, não sendo por isso creditadas e proporcionadas possibilidades de aprendizagem escolar, não fará com que realmente não se aposte no enriquecimento e na optimização das dificuldades (leia-se, competências) das populações com DID, contribuindo assim para a sua exclusão.

Neste sentido, e incorporando a filosofia dos paradigmas mais recentes no contexto das populações especiais, com especial enfoque, nas populações DID, vimos por este meio reforçar a nova proposta para a renomeação de até então "Deficiência Mental/Intelectual" para Dificuldade Intelectual e Desenvolvimental. 
Esta proposta é advogada não só pelas atuais mudanças sentidas e percebidas a nível mundial, como também pela "obrigatoriedade" de se assumir que a palavra-chave nesta matéria seja "dificuldade". Esse palavra oferece a possibilidade de, com os apoios adequados, o sujeito poder adotar o papel que lhe é esperado pelos valores sócio-culturais vigentes, descartando, assim, as concepções de caráter de solidariedade/caridade, descredibilização e pena, que ainda hoje lhe são concebidas com base em preconceitos. A nova nomenclatura deverá ser funcional, diretamente relacionada com os critérios e características específicas e, acima de tudo, deve ser respeitadora das pessoas a quem é diagnosticada.

Com a cada vez maior participação das pessoas com DID e suas famílias, é urgente que as decisões políticas considerem as investigações e paradigmas mais recentes que vem sendo reconhecidos, para uma melhor conceitualização e operacionalização dos resultados que reflitam o valor e o impacto dos serviços e apoios.

De acordo com Verdugo e Schalock (2010), a constante renomeação da terminologia e concepção da DID é um fator positivo, na medida em que reflete que "todos os dias" se desenvolvem progressos na área, aumentando a compreensão sobre a mesma, pelo que a utilização da terminologia mais adequada e o desenvolvimento de boas práticas de avaliação e planificação de apoios é por demais evidente.

Resumindo, Portugal encontra-se num momento de transformação, onde os termos de avaliação e qualidade imperam em todos os sistemas político-sociais, pautando-se toda e qualquer atividade pela "avaliação da sua qualidade", seja ao nível dos serviços, dos apoios, da qualidade de vida... realçando-se, a necessidade da concretização efetiva da participação plena das pessoas com DID, que também devem assumir o seu papel nesta demanda (WALSH, 2002).

De acordo com a revisão da literatura e, apesar de muitos autores poderem inclusive colocar a hipótese de que qualquer terminologia utilizada irá adotar sempre uma função de rotulação (mais tarde ou mais cedo), a idéia, por nós advogada, é que se "acertarmos" o passo entre o novo paradigma e a renomeação da nomenclatura, contribuiremos para uma mudança atitudinal da sociedade em geral face às pessoas com DID! E porque é de pessoas que estamos a falar, essa mudança começa agora... acertando mais falar-se de Dificuldade Intelectual do que de Deficiência Mental.

\section{REFERÊNCIAS}

AMIRALIAN, M. etal. Conceituando deficiência. Revista de Saúde Pública, São Paulo, v.34, n.1, p.97-103, 2000.

BAROFF, G. Mental Retardation: some issues of concern. In: SWITZKY, H.;

GREENSPAN, S.. What is mental retardation? Ideas for evolving disability, AAMR, 2003. p.64-74. 
COSTA, V. Panorama nacional sobre as pessoas com deficiências: desafios e perspectivas. In: MENDES, E.; ALMEIDA, M.; HAYASHI, M. (Org.). Temas em Educação Especial: conhecimento para fundamentar a prática. CAPES - Programa de Apoio à Educação Especial PROESP, Junqueira \& Marin Editores, 2010. p.99-110.

DEPARTMENT OF HEALTH. Valuing People: A new strategy for Learning Disability fot the $21^{\text {st }}$ Century, England, 2001.

DINIZ, D.; MEDEIROS, M.; SQUINCA, F. Reflexões sobre a versão em Português da Classificação Internacional de Funcionalidade, Incapacidade e Saúde", Cadernos de Saúde Pública, Rio de Janeiro, v.23, n.10, p.2507-2510, 2007.

GELB, S. The dignity of humanity is not a scientific construct. Mental Retardation, v.40, n.1, p.55-56, 2002.

GLIDDEN, L. Where are the Mental and Retardation in Mental Retardation. In:

SWITZKY, H.; GREENSPAN, S. What is mental retardation? Ideas for evolving disability, AAMR, 2003. p. 7586

GOODE, D. Mental retardation is dead: long live mental retardation! Symposium: What's in a name? Mental Retardation, v.40, n.1, p.57-59, 2002.

LEONARD, H.; WEN, X.. The Epidemiology of mental retardation: challenges and opportunities in the new millenium, Mental Retardation and Developmental Disabilities Research Reviews, v.8, n.3, p.117-134, 2002.

LUCKASSON, R. et al. Mental Retardation: Definitions, Classification and Systems of Supports. 10th Edition, AAMR, 2002.

LUCKASSON, R.; REEVE, A. Naming, defining and classofying in mental retardation. Mental Retardation. v.39, p.47-52, 2001.

MAZZOTTA, M. Desafios para a Política e a Pesquisa em Educação Especial no Brasil. In: MENDES, E.; ALMEIDA, M.; HAYASHI, M. (Org.). Temas em Educação Especial: conhecimento para fundamentar a prática. CAPES - Programa de Apoio à Educação Especial PROESP/Junqueira \& Marin Editores, 2008. p.79-87.

MORATO, P.; SANTOS, S. Dificuldades intelectuais e desenvolvimentais. A mudança de paradigma na concepção da deficiência mental, Revista de Educação Especial e Reabilitação, IV série, v.14, p. 51-55, 2007.

OMS. Classificação Internacional de Funcionalidade, Incapacidade e Saúde. Organização Mundial de Saúde, Direção Geral de Saúde, Lisboa, 2004.

PANEK, P.; SMITH, J. Assessment of terms to describe mental retardation. Research in Developmental Disabilities, v.26, p.565-576, 2005.

ROSSATO, S.; LEONARDO, N. A deficiência intelectual na concepção de educadores da educação especial: contribuições da psicologia histórico cultural, Revista Brasileira de Educação Especial, v.17, n.1, p.71-86, 2011.

SANTOS, S.; MORATO, P. Comportamento Adaptativo. Porto Editora, 2002. Coleção Educação Especial, n.8. 
DID na DID: Diagnóstico, intervenção e des-institucionalização na dificuldade intelectual e desenvolvimental. SEMINÁRIO INTERNACIONAL SOBRE DEFICIÊNCIA. Como acrescentar valor às organizações que valorizam a diferença. Minho, 2010. Actas... Universidade do Minho, 2010. (no prelo).

SANTOS, S. et al. Adaptação da Escala de Intensidade de Apoios: Estudo Preliminar, Revista de Educação Especial, IV Série, v.15, p.43-64, 2008.

SASSAKI, R. Atualizações semânticas na inclusão das pessoas - deficiência mental ou intelectual? Doença ou transtorno mental? Revista Nacional de Reabilitação, v.9, n.43, p.9-10, 2005.

SCHALOCK, R. Mental Retardation: a condition characterized by significant limitations in practical, conceptual and social skills. in Switzky, H. N. et al, What is Mental Retardation - Ideas for an Evolving Disability. AAMR, USA. 2003. p. 271-283.

SCHALOCK, R. L. et al. Perspectives: The renaming of mental retardation: Understanding the change to the term intellectual disability, Intellectual and Developmental Disabilities. v.45, p.116-124, 2007a.

SCHALOCK, R. et al. User's guide: Mental Retardation - definition, classification and systems of supports, 10th Edition, AAIDD, USA, 2007b

SCHALOCK, R. et al. Intellectual Disability - Definition, Classification ad Systems of supports, 11th Edition, Washington, DC: AAIDD, 2010.

SMITH, J. D., The myth of mental retardation: paradigm shifts, disaggregation and developmental disabilities. symposium: What's in a name? Mental Retardation, v.40, n.1, p.62-64, 2002.

Abandoning the myth of mental retardation. Education and Training in Mental Retardation and Developmental Disabilities, v.38, n.4, p.358-361, 2003.

THOMPSON, J. ET AL. Supports Intensity Scale - Users Manual. American Association on Mental Retardation, 2004.

TURNBULL, R. et al. Shakespeare Redux, or Romeo and Juliet Revisited: Embedding a terminology and name change in a new agenda for the field of mental retardation. Mental Retardation, v.40, n.1, p.65-70, 2002.

VERDUGO, M.; SCHALOCK, R. Últimos advances en el enfoque y concepción de las personas com discapacidad intellectual. Revista Española sobre Discapacidad Intelectual. v.41, n.236, p.7-21, 2010.

WALSH, K. Thoughts on changing the term mental retardation. Symposium: What's in a name? Mental Retardation, v.40, n.1, p.70-75, 2002.

WOLFENSBERGER, W. Needed or at least wanted: sanity in the language wars. symposium: what's in a name. Mental Retardation, v.40, n.1, p.75-80, 2002.

WHO. ICD-10 Guide for Mental Retardation. Geneva, World Health Organization, 1996.

Recebido em: 13/10/2011

Reformulado em: 15/03/2012

Aprovado em: 20/03/2012 